\title{
Wide Genetic Diversity of Blastocystis in White-Tailed Deer (Odocoileus virginianus) from Maryland, USA
}

\author{
Jenny G. Maloney ${ }^{\mathbb{D}}$, Yunah Jang, Aleksey Molokin, Nadja S. George and Monica Santin *(D) \\ Environmental Microbial and Food Safety Laboratory, Agricultural Research Service, \\ United States Department of Agriculture, Beltsville, MD 20705, USA; jenny.maloney@usda.gov (J.G.M.); \\ yunahj73@gmail.com (Y.J.); aleksey.molokin@usda.gov (A.M.); nadja.george@usda.gov (N.S.G.) \\ * Correspondence: monica.santin-duran@usda.gov; Tel.: +1-301-504-6774
}

Citation: Maloney, J.G.; Jang, Y.;

Molokin, A.; George, N.S.; Santin, M. Wide Genetic Diversity of Blastocystis in White-Tailed Deer (Odocoileus virginianus) from Maryland, USA. Microorganisms 2021, 9, 1343.

https://doi.org/10.3390/

microorganisms 9061343

Academic Editor: Eric Viscogliosi

Received: 25 May 2021

Accepted: 14 June 2021

Published: 21 June 2021

Publisher's Note: MDPI stays neutral with regard to jurisdictional claims in published maps and institutional affiliations.

Copyright: (C) 2021 by the authors Licensee MDPI, Basel, Switzerland. This article is an open access article distributed under the terms and conditions of the Creative Commons Attribution (CC BY) license (https:// creativecommons.org/licenses/by/ $4.0 /)$.

\begin{abstract}
Blastocystis is a gastrointestinal protist frequently reported in humans and animals worldwide. Wildlife populations, including deer, may serve as reservoirs of parasitic diseases for both humans and domestic animals, either through direct contact or through contamination of food or water resources. However, no studies of the occurrence and subtype distribution of Blastocystis in wildlife populations have been conducted in the United States. PCR and next generation amplicon sequencing were used to determine the occurrence and subtypes of Blastocystis in white-tailed deer (Odocoileus virginianus). Blastocystis was common, with $88.8 \%(71 / 80)$ of samples found to be positive. Twelve subtypes were identified, ten previously reported (ST1, ST3, ST4, ST10, ST14, ST21, and ST23-ST26) and two novel subtypes (ST30 and ST31). To confirm the validity of ST30 and ST31, MinION sequencing was used to obtain full-length SSU rRNA gene sequences, and phylogenetic and pairwise distance analyses were performed. ST10, ST14, and ST24 were the most commonly observed subtypes. Potentially zoonotic subtypes ST1, ST3, or ST4 were present in $8.5 \%$ of Blastocystis-positives. Mixed subtype infections were common (90.1\% of Blastocystis-positives). This study is the first to subtype Blastocystis in white-tailed deer. White-tailed deer were found to be commonly infected/colonized with a wide diversity of subtypes, including two novel subtypes, zoonotic subtypes, and subtypes frequently reported in domestic animals. More studies in wildlife are needed to better understand their role in the transmission of Blastocystis.
\end{abstract}

Keywords: Blastocystis; MinION; NGS; ribosomal RNA; subtypes; white-tailed deer; USA

\section{Introduction}

Blastocystis sp. is one of the most common protists colonizing/infecting the gastrointestinal tract of humans and numerous animals and has a global distribution [1-4]. The pathogenicity of Blastocystis is controversial, as the presence of Blastocystis in humans has been associated with gastrointestinal symptoms and/or urticaria, but it is also commonly found in asymptomatic individuals [5-7]. It has also been suggested that Blastocystis colonization could be associated with a healthy gut microbiome [4]. Blastocystis transmission is via the fecal-oral route, with direct transmission through contact with infected humans/animals or indirect transmission through ingestion of contaminated food and water [8-11]. The identification of Blastocystis in a broad range of animals, including pets, livestock, and wildlife, indicates that animals could be a potential source of infection for humans [3].

There is considerable genetic diversity among Blastocystis specimens isolated from mammals and birds that has been described based on polymorphisms in the small subunit ribosomal RNA (SSU rRNA) gene [12]. So far, 29 genetic variants, called subtypes (ST), have been proposed, and of those only 25 subtypes meet the current recommended criteria for unique subtype designations (ST1-ST17, ST21, ST23-ST29) [13-16]. Ten subtypes have been reported in humans, ST1-ST9 and ST12, with most studies reporting primarily ST1ST4 [17]. The subtypes reported in humans have also been observed in animals indicating 
that these subtypes may have zoonotic potential [18-22]. For example, the identification of ST6, a subtype mostly identified in avian species, in slaughterhouse staff members provided evidence of the potential zoonotic transmission of this subtype through direct contact between chickens and their handlers [21]. Similarly, ST5, the most common subtype reported in pigs, is only sporadically reported in humans but was present in piggery staff, suggesting that close contact with pigs was associated with zoonotic transmission of ST5 in piggery workers [20].

The multiple transmission routes and the capacity of Blastocystis to infect many avian and mammalian hosts, including wild species, provide the appropriate conditions for transmission between humans and wild and domestic animals [3]. However, few studies on the occurrence and subtypes of Blastocystis in wildlife have been conducted, and there are no studies from the United States [3]. Better knowledge of subtypes of Blastocystis in wildlife is necessary to understand the potential role of wildlife in the transmission dynamics of this organism and the potential role of wildlife as reservoirs for human infection. The wild ungulate communities, including deer populations, frequently occur sympatrically with free-ranging domestic animals and are observed in close proximity to human populations. Blastocystis has been identified from a wide range of wild ungulates [3], but information on the presence and subtype distribution of Blastocystis in deer are limited (Table 1). Previous studies have reported the presence of Blastocystis in wild deer and in deer living in captivity and on farms with significant differences in occurrence between studies. To date, Blastocystis has been documented in 12 deer species, but animal numbers in these surveys tend to be small (Table 1). Furthermore, reported prevalence ranges widely from $0.8 \%$ in farmed sika deer in China [23] up to 100\% in multiple populations including captive white-lipped deer (Cerous albirostris) in China [24], fallow deer from a zoo in Mauritius [25], captive marsh deer (Blastocerus dichotomus) and gray brocket (Mazama gouazoubira) in Brazil [26], and muntjac deer, rein deer, and red deer from a zoo in the United Kingdom [27,28]. There are no published reports of testing for Blastocystis in any deer species in the United States and no studies in white-tailed deer (Odocoileus virginianus) (WTD) worldwide. In the state of Maryland, the white-tailed deer population is estimated at 240,000 deer, with the population increasing on an average of $12 \%$ every 5 years, mostly due to the conversion of forested and agricultural lands into suburban areas that improve habitat conditions for deer (MD-Annual-Deer-Report-2019-2020.pdf (maryland.gov); accessed on 4 May 2021). WTD populations in Maryland live near or in suburban areas where they frequently encounter humans, companion animals, and livestock. The present study was conducted to provide the first examination of the presence of Blastocystis in WTD from Maryland (USA) and to characterize subtypes present in samples using next generation amplicon sequencing (NGS). Additionally, Oxford Nanopore MinION long-read sequencing was used to generate the full-length SSU rRNA gene of two novel Blastocystis sequences identified in WTD to confirm the validity of these sequences as novel subtypes.

Table 1. Summary of studies reporting prevalence and subtypes of Blastocystis identified in deer. Potentially zoonotic subtypes are in bold.

\begin{tabular}{|c|c|c|c|c|c|}
\hline Host (Scientific Name) & Country & $\begin{array}{l}\text { Source of } \\
\text { Deer }\end{array}$ & $\begin{array}{c}\text { No. of Samples } \\
\text { Examined/ } \\
\text { No. of Positives (\%) }\end{array}$ & Subtype(s) & References \\
\hline \multirow{2}{*}{ Eurasia elk (Alces alces) } & United Kingdom & Zoo & $2 / 1(50 \%)^{\mathrm{a}}$ & ST4(1), ST10(1), ST14(1), ND(1) & [27] \\
\hline & United Kingdom & Zoo & $3 / 1(33.3 \%)^{a}$ & ST4(1), ST10(1), ST14(4) & [28] \\
\hline \multirow{2}{*}{ Fallow deer (Dama dama) } & China & Captive & $2 / 1(50 \%)$ & ST10(1) & [24] \\
\hline & Mauritius & Zoo & $2 / 2(100 \%)$ & ST10(2) & [25] \\
\hline Gray brocket (Mazama gouazoubira) & Brazil & Captive & $1 / 1(100 \%)$ & ST5(1) & [26] \\
\hline $\begin{array}{l}\text { Korean water deer (Hydropotes inermis } \\
\text { argyropus) }\end{array}$ & South Korea & Wild & $125 / 51(40.8 \%)$ & ST4(1), ST14(25) ${ }^{\mathrm{b}}$ & [29] \\
\hline Marsh deer (Blastocerus dichotomus) & Brazil & Captive & $1 / 1(100 \%)$ & $\mathrm{ND}(1)^{\mathrm{b}}$ & [26] \\
\hline \multirow{2}{*}{ Muntjac deer (Muntiacus reevesi) } & United Kingdom & Zoo & $1 / 1(100 \%)$ & ST14(1) & [27] \\
\hline & United Kingdom & Zoo & $1 / 1(100 \%)$ & ST13(1) & [28] \\
\hline
\end{tabular}


Table 1. Cont.

\begin{tabular}{|c|c|c|c|c|c|}
\hline Host (Scientific Name) & Country & $\begin{array}{c}\text { Source of } \\
\text { Deer }\end{array}$ & $\begin{array}{c}\text { No. of Samples } \\
\text { Examined/ } \\
\text { No. of Positives (\%) }\end{array}$ & Subtype(s) & References \\
\hline \multirow{5}{*}{ Red deer (Cervus elaphus) } & Australia & Wild & $50 / 1(2 \%)$ & ST4(1) & [30] \\
\hline & China & Captive & $3 / 1(33.3 \%)$ & ST10(1) & [24] \\
\hline & China & Zoo & $5 / 2(40 \%)$ & ST10(2) & [31] \\
\hline & United Kingdom & Zoo & $1 / 1(100 \%)^{a}$ & ST4(2), ST10(6) & [27] \\
\hline & United Kingdom & Zoo & $3 / 1(33.3 \%)^{a}$ & ST4(3), ST10(5) & [28] \\
\hline \multirow{2}{*}{ Reindeer (Rangifer tarandus) } & China & Farm & $104 / 7(6.7 \%)$ & ST10(3), ST13(4) & [23] \\
\hline & United Kingdom & Zoo & $1 / 1(100 \%)$ & ST10(1) & [28] \\
\hline \multirow{2}{*}{ Roe deer (Capreolus capreolus) } & Denmark & Zoo & $1^{c}$ & ST10(1) & [32] \\
\hline & United Kingdom & Zoo & $2 / 1(50 \%)$ & ST5(1) & [25] \\
\hline \multirow{4}{*}{ Sika deer (Cervus nippon) } & China & Farm & $6 / 760(0.8 \%)$ & ST10(5), ST14(1) & [33] \\
\hline & China & Captive & $8 / 3(37.5 \%)$ & ST10(3) & [24] \\
\hline & China & Farm & $82 / 12(14.6 \%)$ & ST10(10), ST14(2) & [23] \\
\hline & China & Zoo & $11 / 1(9.1 \%)$ & ST1(1) & [4] \\
\hline Spotted deer (Axis axis) & Bangladesh & Zoo & $30 / 1(3.3 \%)$ & ST14(1) & [34] \\
\hline White-lipped deer (Cervus albirostris) & China & Captive & $1 / 1(100 \%)$ & ST10(1) & [24] \\
\hline White tailed-deer (Odocoileus virginianus) & United States & Wild & $80 / 71(88.8)$ & $\begin{array}{c}\text { ST1(1), ST3(2), ST4(3), } \\
\text { ST10(51), ST14(30), ST21(14), } \\
\text { ST23(14), ST24(55), ST25(1), } \\
\text { ST26(2), ST30(11), ST31(19)d }\end{array}$ & $\begin{array}{l}\text { This } \\
\text { study }\end{array}$ \\
\hline
\end{tabular}

ND: Not determined. ${ }^{\text {a }}$ The number of STs identified is based on the number of sequence positive clones obtained and does not correspond with the number of positive samples identified. ${ }^{b}$ Not all PCR positive samples were successfully sequenced. ${ }^{c}$ This study was not a survey. ${ }^{d}$ The numbers of STs exceed the number of samples because multiple subtypes were observed in individual samples. For details of ST combinations, see Table 2.

Table 2. Blastocystis prevalence and subtypes observed in white-tailed deer (WTD) in Maryland, USA by age and gender. Potentially zoonotic subtypes are in bold.

\begin{tabular}{|c|c|c|c|c|c|}
\hline & & $\begin{array}{l}\text { No. of } \\
\text { WTD }\end{array}$ & $\begin{array}{l}\text { No. Blas- } \\
\text { tocystis } \\
\text { Positives } \\
\quad(\%)\end{array}$ & Subtypes Identified & Subtypes Combinations Observed in Individual Samples \\
\hline \multirow{3}{*}{$\begin{array}{c}\text { Age } \\
\text { group }^{a}\end{array}$} & Fawn & 3 & $3(100)$ & $\begin{array}{l}\text { ST10, ST14, ST21, } \\
\text { ST23, ST24, ST30 }\end{array}$ & ST10/ST14/ST21(1); ST14/ST21/ST23(1); ST10/ST14/ST21/ST23/ST24/ST30(1) \\
\hline & Yearling & 22 & $19(86.4)$ & $\begin{array}{l}\text { ST3, ST10, ST14, } \\
\text { ST23, ST24, ST26, } \\
\text { ST30, ST31 }\end{array}$ & $\begin{array}{c}\text { ST3/ST31(1); ST3/ST10/ST14/ST24(1); ST10/ST14/ST24(2); ST10/ST24(4); } \\
\text { ST10/ST24/ST26(1); ST10/ST23/ST24(1); ST10/ST30(1); ST10/ST31(2); ST10/ST24/ST31(2); } \\
\text { ST14/ST24(2); ST24(1); ST24/ST31(1) }\end{array}$ \\
\hline & Adult & 54 & $48(88.9)$ & $\begin{array}{l}\text { ST1, ST4, ST10, ST14, } \\
\text { ST21, ST23, ST24, } \\
\text { ST25, ST26, ST30, } \\
\text { ST31 }\end{array}$ & $\begin{array}{l}\text { ST1/ST10/ST21/ST24(1); ST4/ST24(1); ST4/ST24/ST31(1); } \\
\text { ST4/ST10/ST14/ST21/ST24/ST25/ST26(1); ST10/ST14(2); ST10/ST14/ST23/ST24(1); } \\
\text { ST10/ST14/ST24(5);ST10/ST14/ST31 (1);ST10/ST14/ST21/ST24/ST30(3); } \\
\text { ST10/ST14/ST21/ST23/ST24(2); ST10/ST14/ST21/ST23/ST24/ST30(2); } \\
\text { ST10/ST14/ST23/ST24/ST30(2);ST10/ST14/ST24/ST31(1); ST10/ST31(2); } \\
\text { ST10/ST21/ST24(2); ST10/ST23(2); ST10/ST23/ST24(1); ST10/ST24(4);ST10/ST24/ST30(1); } \\
\text { ST10/ST24/ST31(1);ST14(1);ST14/ST24(1);ST24(3);ST24/ST30(1);ST24/ST31(4); ST31(2) }\end{array}$ \\
\hline \multirow{2}{*}{$\underset{\mathrm{a}}{\text { Gender }}$} & Males & 36 & $31(86.1)$ & $\begin{array}{l}\text { ST3, ST4, ST10, ST14, } \\
\text { ST21, ST23, ST24, } \\
\text { ST25, ST26, ST30, } \\
\text { ST31 }\end{array}$ & $\begin{array}{c}\text { ST3/ST31(1); ST3/ST10/ST14/ST24(1); ST4/ST24(1); ST4/ST24/ST31(1); } \\
\text { ST4/ST10/ST14/ST21/ST24/ST25/ST26(1);ST10/ST14/ST23/ST24(2); } \\
\text { ST10/ST14/ST24(4); ST10/ST14/ST21/ST24/ST30(1); ST10/ST14/ST21/ST23/ST24(1); } \\
\text { ST10/ST14/ST23/ST24/ST30(1); ST10/ST14/ST24/ST31(1); ST14/ST21/ST23(1); } \\
\text { ST10/ST30(1); ST10/ST31(1); ST10/ST23(1);ST10/ST24(3);ST10/ST24/ST26(1); } \\
\text { ST10/ST24/ST31(2);ST14/ST24(2);ST24(2);ST24/ST30(1);ST24/ST31(2) }\end{array}$ \\
\hline & Females & 43 & $39(90.7)$ & $\begin{array}{l}\text { ST1, ST10, ST14, } \\
\text { ST21, ST23, ST24, } \\
\text { ST30, ST31 }\end{array}$ & $\begin{array}{l}\text { ST1/ST10/ST21/ST24(1); ST10/ST14(2); ST10/ST14/ST21(1); ST10/ST14/ST24(3); } \\
\text { ST10/ST14/ST31 (1); ST10/ST14/ST21/ST24/ST30(2); ST10/ST14/ST21/ST23/ST24(1); } \\
\text { ST10/ST14/ST21/ST23/ST24/ST30(3); ST10/ST14/ST23/ST24/ST30(1); ST10/ST31(3); } \\
\text { ST10/ST21/ST24(2); ST10/ST23(1); ST10/ST23/ST24(2); ST10/ST24(5); ST10/ST24/ST30(1); } \\
\text { ST10/ST24/ST31(1);ST14(1); ST14/ST24(1);ST24(2); ST24/ST31(3);ST31(2) }\end{array}$ \\
\hline Total & & 80 & $71(88.8)$ & $\begin{array}{l}\text { ST1, ST3, ST4, ST10, } \\
\text { ST14, ST21, ST23, } \\
\text { ST24, ST25, ST26, } \\
\text { ST30, and ST31 }\end{array}$ & $\begin{array}{l}\text { ST1/ST10/ST21/ST24(1); ST3/ST10/ST14/ST24(1); ST3/ST31(1); ST4/ST24(1); } \\
\text { ST4/ST24/ST31(1); ST4/ST10/ST14/ST21/ST24/ST25/ST26(1); ST10/ST14(2); } \\
\text { ST10/ST14/ST23/ST24(1);ST10/ST14/ST24(7); ST10/ST14/ST31 (1); } \\
\text { ST10/ST23/ST24/ST31(1); ST10/ST14/ST21(1); ST10/ST14/ST21/ST23/ST24(2); } \\
\text { ST10/ST14/ST21/ST24/ST30(3);ST10/ST14/ST21/ST23/ST24/ST30(3); } \\
\text { ST10/ST14/ST23/ST24/ST30(2);ST10/ST14/ST24/ST31(1);ST10/ST30(1); ST10/ST31(4); } \\
\text { ST14/ST21/ST23(1);ST10/ST21/ST24(2);ST10/ST23(2); ST10/ST23/ST24(2); ST10/ST24(8); } \\
\text { ST10/ST24/ST26(1); ST10/ST24/ST30(1);ST10/ST24/ST31(3);ST14(1); ST14/ST24(3); } \\
\text { ST24(4);ST24/ST30(1);ST24/ST31(5); ST31(2) }\end{array}$ \\
\hline
\end{tabular}

\footnotetext{
${ }^{\text {a }}$ No gender and age information available for 1 WTD.
} 


\section{Materials and Methods}

\subsection{Source and Collection of Specimens}

Feces were collected from 80 hunter-killed WTD during a managed hunt in Howard County, Maryland. The hunt was conducted under the supervision of county officials as part of a wildlife management program. The goals of the management program are to ensure the present and future well-being of deer and their habitat and to maintain the deer population at a level that safeguards compatibility with human land uses and natural communities. Fecal specimens were collected over two hunting seasons: 2010-2011 $(n=52)$ and 2011-2012 $(n=28)$. Age and gender data were recorded for each WTD sampled (Table 2). A fecal specimen was collected from each animal directly from the rectum into a plastic cup. Cups were capped, labeled, and immediately placed in an insulated container packed with ice or cold packs. Specimens were transported to the USDA laboratory in Beltsville, Maryland and processed within 1-3 days of collection.

\subsection{Parasite Concentration from Feces and DNA Extraction}

To concentrate parasites, fecal specimens were sieved and subjected to $\mathrm{CsCl}$ density centrifugation, as previously described [35]. DNA was extracted from each CsClconcentrated fecal sample using the DNeasy Tissue Kit (Qiagen, Valencia, CA, USA) as directed by the manufacturer with minor modifications. In brief, a $50 \mu \mathrm{L}$ suspension of each CsCl-concentrated fecal sample was suspended in $180 \mu \mathrm{L}$ of ATL buffer and thoroughly mixed. Twenty $\mu \mathrm{L}$ of proteinase $\mathrm{K}(20 \mathrm{mg} / \mathrm{mL})$ was added to this suspension, and the mixture was incubated at $55^{\circ} \mathrm{C}$ overnight. Then $200 \mu \mathrm{L}$ of AL buffer was added, and DNA was purified per manufacturer's instructions and eluted in $100 \mu \mathrm{L}$ of AE buffer.

\subsection{Molecular Detection and Subtype Identification Using Next Generation Amplicon Sequencing}

A next generation amplicon sequencing strategy was used to detect Blastocystis as previously described [36]. Briefly, a PCR using primers ILMN_Blast505_532F and ILMN_Blast998_1017R was used to screen all 80 WTD samples. These primers amplify a fragment of the SSU rRNA gene (ca. 500 bp) and are identical to Blast505_532F/Blast998_1017R [37], with the exception of containing the Illumina overhang adapter sequences on the $5^{\prime}$ end. Final libraries were quantified by Qubit fluorometric quantitation (Invitrogen, Carlsbad, CA, USA) prior to normalization. A final pooled library concentration of $8 \mathrm{pM}$ with $20 \%$ PhiX control was sequenced using Illumina MiSeq 600 cycle v3 chemistry (Illumina, San Diego, CA, USA). Paired end reads were processed and analyzed with an in-house pipeline that uses the BBTools package v38.82 [38], VSEARCH v2.15.1 [39], and BLAST+ 2.11.0 [40]. Briefly, read pairs were merged, filtered for quality and length, denoised, and checked for chimeric sequences. Clustering and the assignment of centroid sequences to operational taxonomic units (OTU) was performed within each sample at a 98\% identity threshold. Only those OTUs with a minimum of 100 sequences were retained and then checked for chimeras once more. OTUs were then blasted against Blastocystis references from NCBI. All hits below an alignment length of $400 \mathrm{bp}$ were removed. All raw fastq files were deposited to the NCBI sequence read archive under accession numbers SRR14607063-SRR14607133. The nucleotide sequences generated using NGS in this study were deposited in GenBank under the accession numbers MZ267636-MZ267676.

\subsection{PCR Amplification and Sequencing of the Full-Length SSU rRNA Gene}

For four WTD samples (\#22, \#27, \#73, \#79) containing novel subtypes (ST30 and ST31), we used a previously described Nanopore sequencing strategy to generate the approximately 1800 base pair SSU rRNA gene [41]. Briefly, a PCR using SSU-F1 (5'-AAC CTG GTT GAT CCT GCC AGT AGT C-3') and SSU-R1 (5'-TGA TCC TTC TGC AGG TTC ACC TAC G-3'), which amplify the SSU rRNA gene of most eukaryotic organisms, was performed [42]. Each reaction used $1 \mu \mathrm{M}$ forward and reverse primers and $12.5 \mu \mathrm{L}$ of KAPA HiFi HotStart ReadyMix (KAPABioSystems, Cape Town, South Africa) in a $25 \mu \mathrm{L}$ reaction volume. Initial denaturation was performed at $98^{\circ} \mathrm{C}$ for $5 \mathrm{~min}$ followed by 
35 cycles of amplification $\left(20 \mathrm{~s}\right.$ at $98^{\circ} \mathrm{C}, 45 \mathrm{~s}$ at $60^{\circ} \mathrm{C}$, and $90 \mathrm{~s}$ at $72{ }^{\circ} \mathrm{C}$ ) and final extension for 5 min at $72^{\circ} \mathrm{C}$. Following amplification, amplicons were visualized using a QIAxcel (Qiagen, Valencia, CA, USA) and quantified using a Qubit fluorometer (ThermoFisher Scientific, Waltham, MA, USA).

The Nanopore sequencing library was prepared using the Oxford Nanopore Technologies (ONT) SQK-LSK109 Ligation Sequencing Kit (ONT, Oxford, UK) following the manufacturer's protocol for Amplicons by Ligation (ACDE_9064_v109_revQ_14Aug2019). Amplicons were quantified and diluted to ensure $150 \mathrm{fmol}$ of DNA was used as input into library prep as recommended by the protocol. The nanopore library was run on an R9.4 flow cell (FLO-MIN106) using an ONT MinION Mk1B and MinKNOW v20.06.15 software (ONT, Oxford, UK). Basecalling was performed using Guppy v4.0.11 (ONT, Oxford, UK) using a minimum quality score cut off of 7 for filtering low quality reads. FASTQ reads were also length filtered to only include reads between 1700 and 2000 nucleotides. Reads were then corrected using Canu v2.1 [43] and consensus sequences were generated by clustering reads using the vsearch-cluster_fast command (vsearch v2.14.1) with a 98\% identity threshold, checked for chimeras, and polished as previously described [40].

For comparison purposes, full-length sequences and partial sequences obtained with MinION and MiSeq, respectively, were aligned using ClustalW in MegAlign 15 (DNASTAR Lasergene 15, Madison, WI, USA), and pairwise distances between consensus sequences were calculated. The full-length nucleotide sequences generated in this study were deposited in GenBank under the accession numbers MZ267674-MZ267679.

\subsection{Phylogenetic and Pairwise Distance Analysis}

The full-length SSU rRNA gene nucleotide sequences obtained in this study, appropriate full-length Blastocystis reference nucleotide sequences obtained from the reference database found at http:/ / entamoeba.1shtm.ac.uk/blastorefseqs.htm (accessed on 4 May 2021), as well as other full-length sequences available in GenBank to include all currently accepted subtypes were aligned to generate a phylogenetic tree which was rooted using Proteromonas lacertae, a Stramenopile which is closely related to Blastocystis, as an outgroup. Nucleotide sequences were aligned with the Clustal $\mathrm{W}$ algorithm and the phylogenetic analysis was performed using the Neighbor-Joining (NJ) method, and genetic distances calculated with the Kimura 2-parameter model using MEGA X [44,45]. A total of 1950 positions were included in the final dataset, which included 70 nucleotide sequences. Bootstrapping with 1000 replicates was used to determine support for the clades generated. Additionally, evolutionary analysis was conducted to establish divergence between nucleotide sequences (pairwise distance) using the Kimura 2-parameter model in MEGA X.

Furthermore, for comparative purposes, identical phylogenetic and pairwise distance analyses using the same 70 nucleotide sequences utilized for full-length analyses were conducted for the regions of the SSU rRNA gene amplified by the two most common standard primers sets used for Blastocystis in survey studies to amplify and sequence the regions known as barcoding and Santin $[37,46]$. There was a total of 590 and 570 positions in the final datasets for barcoding and Santin regions, respectively.

\section{Results}

\subsection{Prevalence of Blastocystis in White-Tailed Deer}

Of the 80 WTD fecal samples tested in this study, $71(88.8 \%)$ were determined to be positive for Blastocystis by PCR. Positive-Blastocystis samples were observed in all age groups (Table 2). The highest prevalence was found in fawns $(100 \% ; 3 / 3)$ follow by adults $(88.9 \% ; 48 / 54)$ and yearlings $(86.4 \% ; 19 / 22)$. Blastocystis was detected at similar levels in males $(86.1 \% ; 31 / 36)$ and females $(90.7 \% ; 39 / 43)$.

\subsection{Subtypes of Blastocystis in White-Tailed Deer}

The 71 Blastocystis-positive samples generated a total of $13,785,248$ paired end reads. Following end trimming, quality filtering, and pair merging, 5,006,876 reads remained. 
The removal of chimeric sequences left 3,955,512 merged reads, which were used for OTU generation. Clustering generated 230 OTUs that aligned to Blastocystis among the 71 samples, and of those 38 were unique Blastocystis sequences (Table 3).

Table 3. Blastocystis subtypes identified in white-tailed deer (WTD) from Maryland (USA) including number of samples in which each subtype was identified and number of unique sequences among each subtype.

\begin{tabular}{cccc}
\hline Blastocystis Subtype & $\begin{array}{c}\text { No. of Blastocystis-Positive } \\
\text { Samples }\end{array}$ & $\begin{array}{c}\text { Percentage of Positive WTD } \\
\text { of Unique Sequences } \\
\text { within Subtype }\end{array}$ & $\begin{array}{c}\text { Soly } \\
\text { ST1 }\end{array}$ \\
ST3 & 2 & 1.4 & 2 \\
ST4 & 3 & 2.8 & 2 \\
ST10 & 51 & 4.2 & 2 \\
ST14 & 30 & 71.8 & 3 \\
ST21 & 14 & 42.3 & 3 \\
ST23 & 14 & 19.7 & 1 \\
ST24 & 55 & 19.7 & 3 \\
ST25 & 1 & 77.5 & 1 \\
ST26 & 2 & 1.4 & 3 \\
ST30 & 11 & 2.8 & 3 \\
ST31 & 19 & 15.5 & 1 \\
\hline
\end{tabular}

Twelve subtypes were identified in Blastocystis-positive WTD, ten previously reported subtypes ST1, ST3, ST4, ST10, ST14, ST21, ST23, ST24, ST25, ST26, and two novel subtypes named ST30 and ST31 (Tables 2 and 3). The most frequently observed subtypes in this study were ST10 and ST24 found in 71.8\% (51/71) and 77.5\% (55/71) of Blastocystis-positive WTD, respectively (Table 3). ST14 was the third most abundant subtype and was detected in $42.3 \%$ (30/71) of the Blastocystis-positive WTD. Novel subtypes ST30 and ST31 were frequently observed and were detected in 15.5\% (11/71) and 26.8\% (19/71) of the Blastocystis-positive WTD, respectively (Table 3). Potentially zoonotic subtypes ST1, ST3, and ST4 were observed in one, two, and three samples, respectively (Table 3). An increase in the number of subtypes associated with age was observed, with six subtypes in fawns, eight in yearlings, and 11 in adults (Table 2). Eleven and eight subtypes were identified in males and females, respectively (Table 2).

Mixed infections with two or more Blastocystis subtypes were frequently observed. Infections with more than one subtype were identified in $90.1 \%(64 / 71)$ of Blastocystispositive WTD, while single infections were only detected in $9.9 \%(7 / 71)$ of Blastocystispositive WTD. Only three subtypes were present as single infections, ST14, ST24, and ST31, which were found in $1.4 \%(1 / 71), 5.6 \%(4 / 71)$, and 2.8\% (2/71) of the Blastocystis-positive WTD, respectively (Table 2; Figure 1). Thirty combinations of subtypes were observed in Blastocystis-positive samples including co-infection with two, three, four, five, six, and seven subtypes found in ten, ten, five, three, one, and one samples respectively (Table 2). The relative abundance of subtypes present in each of the 71 Blastocystis-positive samples varied widely (Figure 1). Co-infection with ST10/ST24 and ST10/ST14/ST24 were the most common mixed subtype combinations observed, and they represented $12.5 \%(8 / 64)$ and $10.9 \%(7 / 64)$ of mixed subtype infections, respectively. 


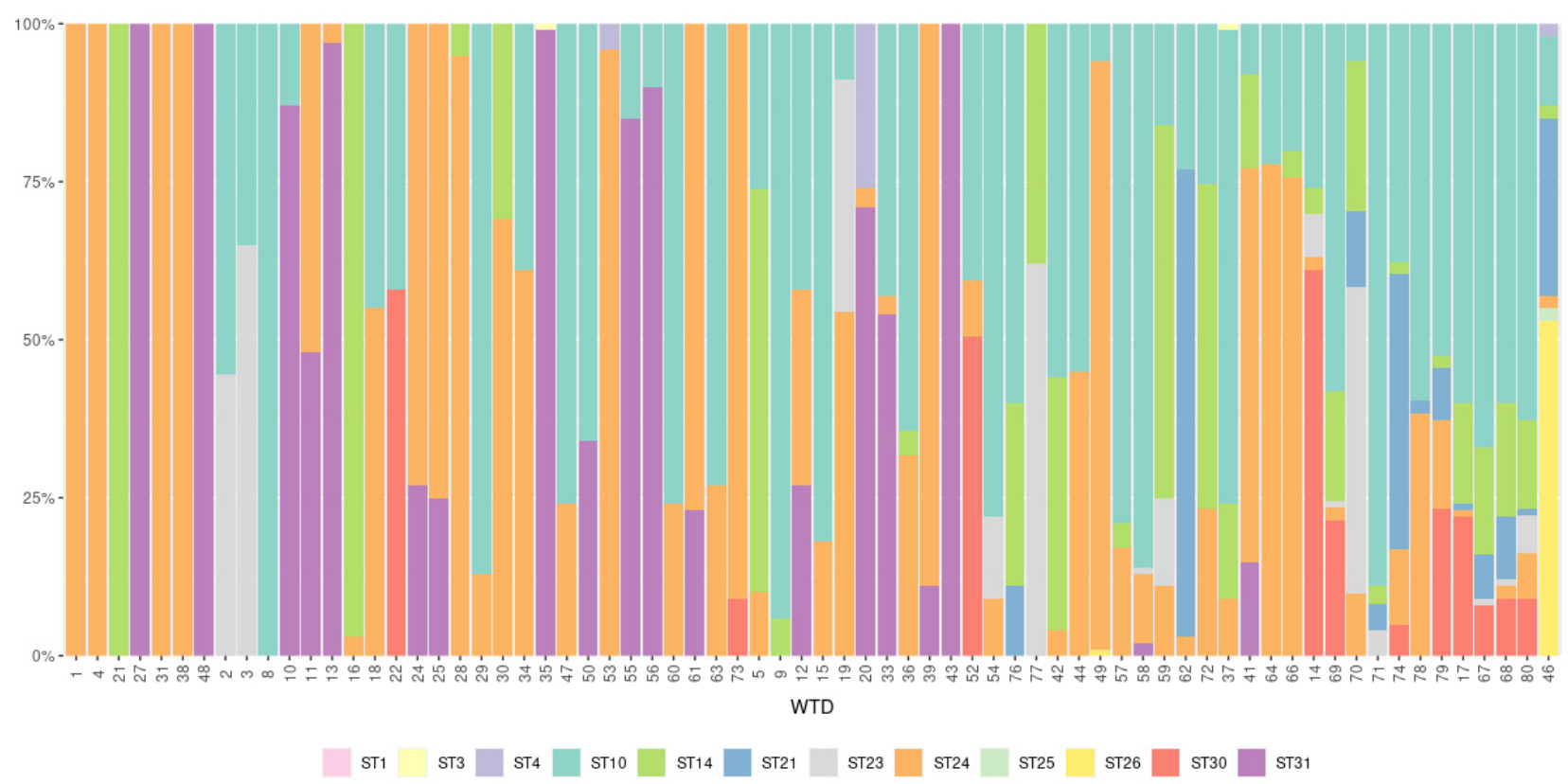

Figure 1. Relative abundance of reads (\%) of each subtype present in the 71 Blastocystis-positive white-tailed deer (WTD) samples arranged by number of subtypes present from lowest to highest.

\subsection{Blastocystis Intra-Subtype Variation}

Thirty-eight unique Blastocystis sequences were identified in the 71 Blastocystis-positive WTD (Table 3). High intra-subtype variation was observed for ST10 with 14 unique sequences among the 51 ST10-positive WTD. Intra-subtype variation was lower for the rest of the subtypes identified in WTD, with three unique sequences for ST14, ST21, ST24, ST26, and ST30, two unique sequences for ST1, ST3, and ST4, and a single unique sequence for ST23, ST25, and ST31 (Table 3).

\subsection{Validation of Novel Subtypes ST30 and ST31}

Nucleotide sequences for the novel subtypes generated by Illumina were compared to nucleotide sequences available in the GenBank database. The closest match to Blastocystis sequences available in GenBank for ST30 was $99.8 \%-100 \%$ to unpublished Blastocystis nucleotide sequences with no subtype information that were obtained from sheep fecal samples from Belgium (HF569206). For ST31, the closest nucleotide sequences available in GenBank were three unpublished Blastocystis nucleotide sequences also without subtype information and that were obtained from fecal samples of Korean water deer from South Korea (MT114839, MT114842, MT114845) with 97.7\% similarity. To confirm the validity of the novel subtypes according to recently proposed guidelines, we used a Nanopore sequencing strategy to obtain the near full-length nucleotide sequence of the SSU rRNA gene using DNA from four WTD (\#22, \#27, \#73, \#79) positive for novel subtype sequence variants. Full-length sequences were successfully obtained for both novel subtypes and for all three variants of ST30. Full-length nucleotide sequences of the SSU rRNA gene for the three sequence variants of ST30 were obtained from WTD\#22, WTD\#73, and WTD\#79, while ST31 was obtained from WTD\#27. Additionally, full-length sequences were generated for other subtypes present in WTD\#22 and WTD\#79. A full-length ST10 sequence was obtained from WTD\#22, and from WTD\#79, individual sequences for ST10, ST21, and ST24 were obtained. ST21 and ST24 full-length sequences from WTD\#79 have been previously published (MW887929 and MW887930) [16]. There was 100\% agreement between the Illumina sequence and the same region within the MinION sequence for all sequences of ST30 and ST31.

Phylogenetic analysis of full-length sequences using the NJ method demonstrated that all 3 variants of the ST30 cluster with ST21 and ST26 with bootstrap support of 68 for the 
cluster formed by ST30 and ST21, and bootstrap support of 100 for the cluster formed by ST30/ST21 and ST26 (Figure 2). Similar clustering is observed the barcoding and Santin regions, with ST30 clustering with ST21 and ST26. Bootstrap values of 75 and 99 were observed for the cluster formed by ST30 and ST21 and 98 and 76 for ST30/ST21 and ST26 for the Barcoding and Santin regions, respectively (Figures 3 and 4). For ST31, phylogenetic analysis using full-length sequences showed ST31 clustering with ST13 with bootstrap support of 94 (Figure 2). Similar clustering is observed using the barcoding region with bootstrap support of 89 (Figure 3). For the Santin region, ST31 no longer forms a clade with ST13, but does cluster within a clade formed by ST12, ST13, ST14, ST24, and ST25 (Figure 4).

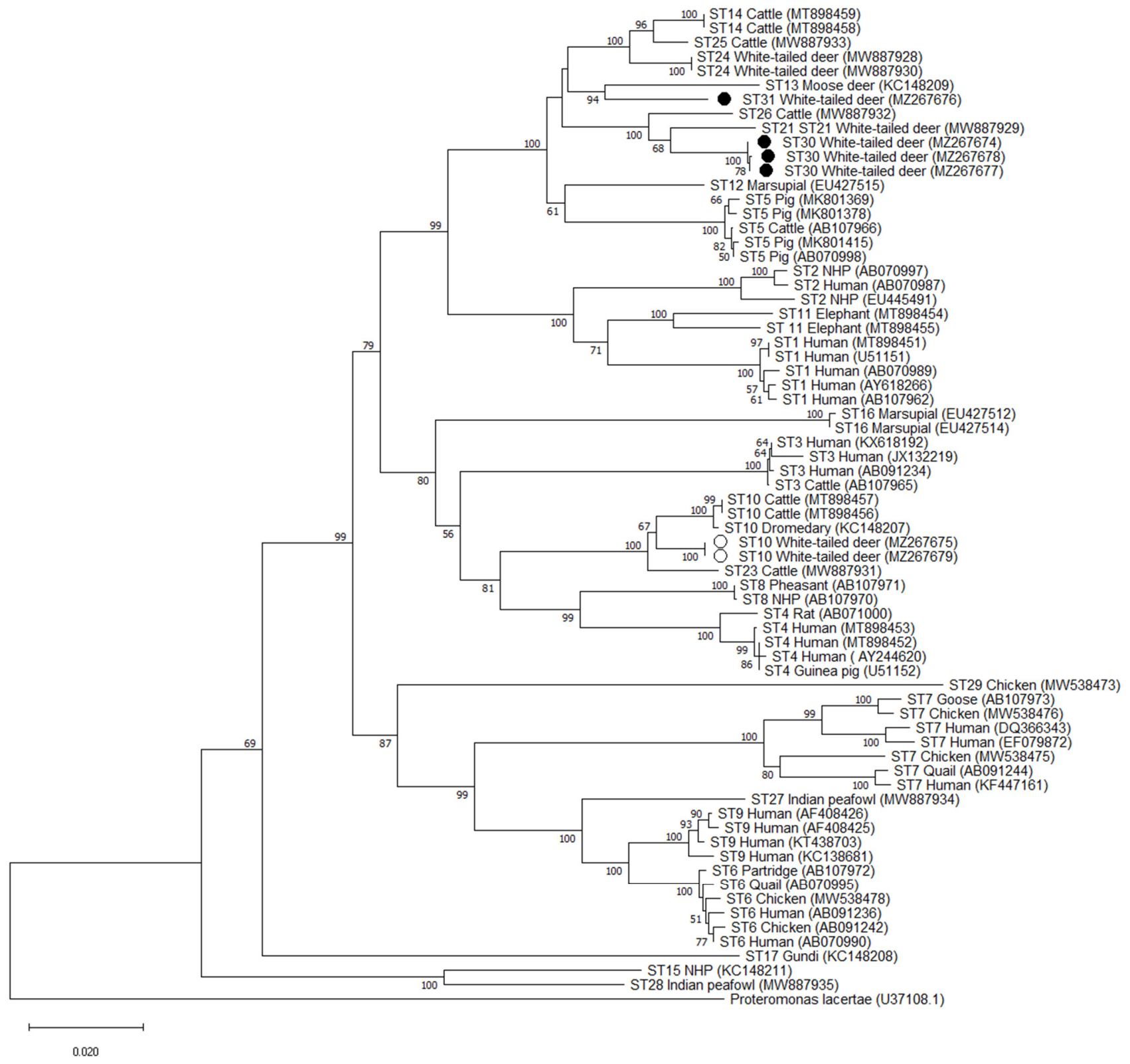

Figure 2. Phylogenetic relationships among Blastocystis full-length sequences generated in the present study (novel subtypes are represented with a black filled circle and other subtypes with an unfilled circle) and representative reference sequences of all accepted subtypes. Proteromonas lacertae was used as outgroup taxon to root the tree. Analysis was conducted by a neighbor-joining method. Genetic distances were calculated using the Kimura two-parameter model. This analysis involved 70 nucleotide sequences, and there were a total of 1950 positions in the final dataset. Bootstrap values lower than $50 \%$ are not displayed. 


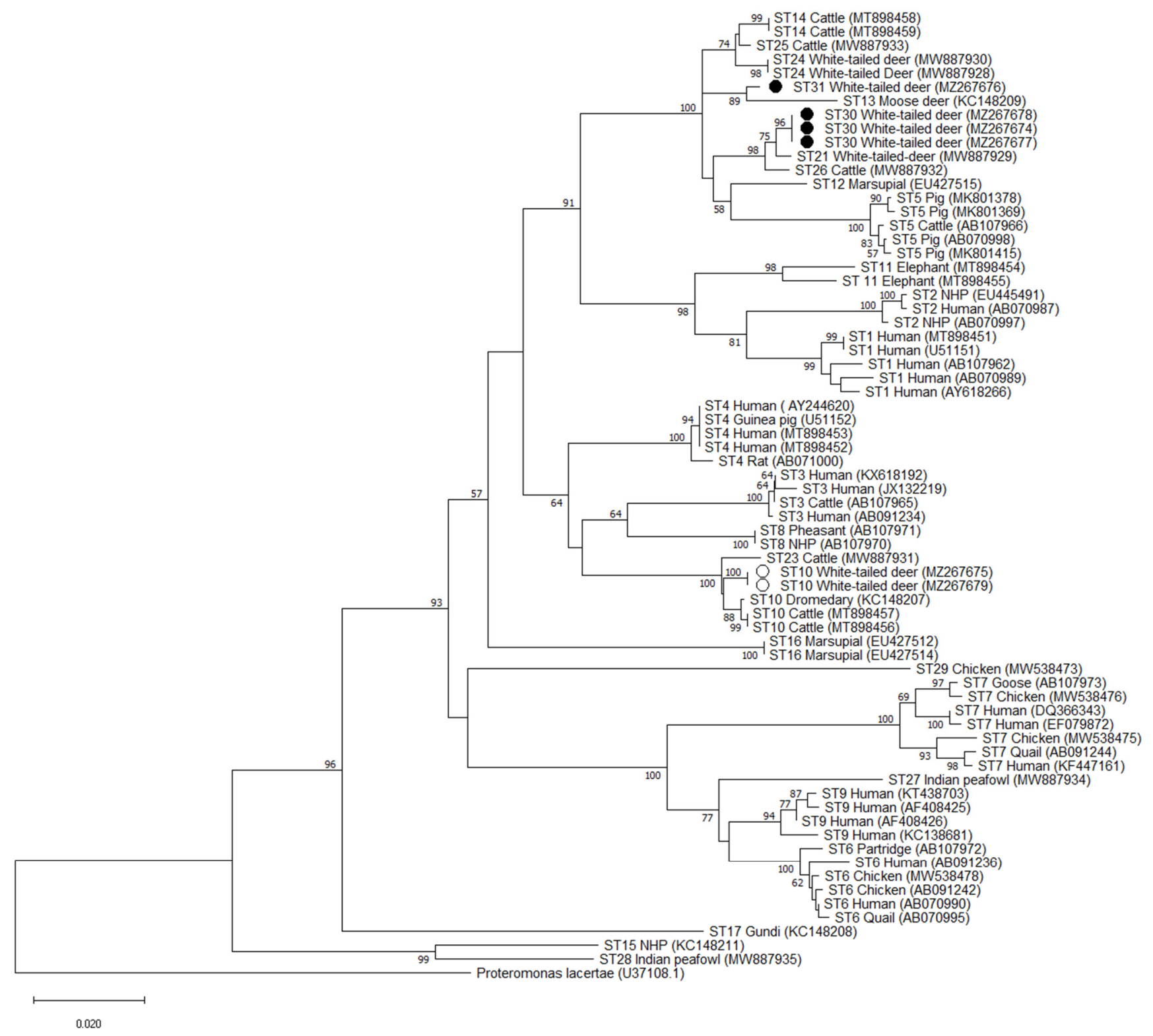

Figure 3. Phylogenetic relationships among Blastocystis barcoding region sequences generated in the present study (novel subtypes are represented with a black filled circle and other subtypes with an unfilled circle) and representative reference sequences of all accepted subtypes. Proteromonas lacertae was used as outgroup taxon to root the tree. Analysis was conducted by a neighbor-joining method. Genetic distances were calculated using the Kimura two-parameter model. This analysis involved 70 nucleotide sequences, and there were a total of 590 positions in the final dataset. Bootstrap values lower than $50 \%$ are not displayed. 


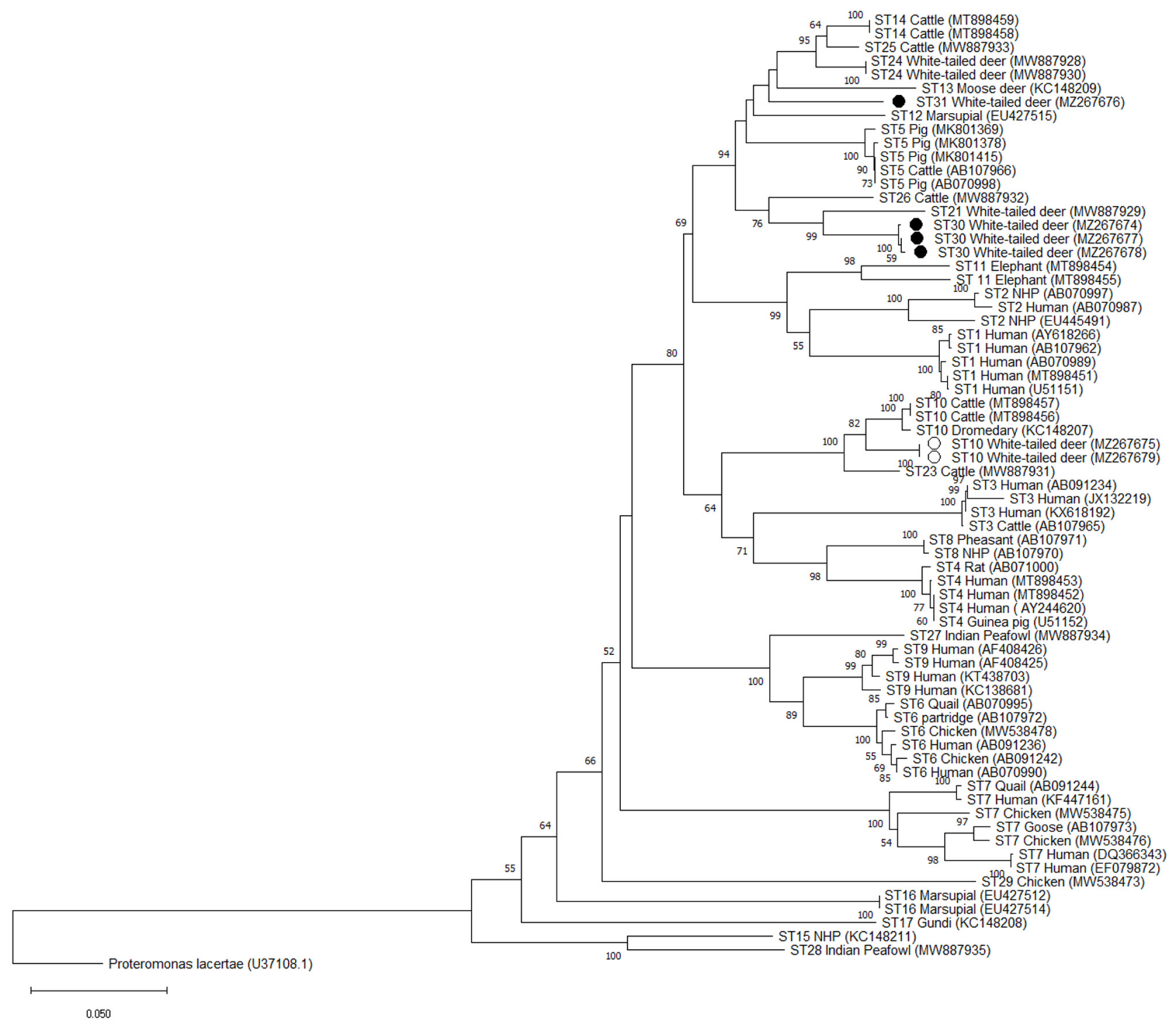

Figure 4. Phylogenetic relationships among Blastocystis Santin region sequences generated in the present study (novel subtypes represented are with a black filled circle and other subtypes with an unfilled circle) and representative reference sequences of all accepted subtypes. Proteromonas lacertae was used as outgroup taxon to root the tree. Analysis was conducted using a neighbor-joining method. Genetic distances were calculated using the Kimura two-parameter model. This analysis involved 70 nucleotide sequences, and there were a total of 571 positions in the final dataset. Bootstrap values lower than $50 \%$ are not displayed.

Pairwise distance comparisons were used to evaluate the percentage of shared sequence identity of ST30 and ST31 with known subtypes using full-length sequences, the barcoding region, and the Santin region (Supplementary Tables S1-S3). Using full-length sequences, the highest percentage of sequence similarity for ST30 was $97 \%$ with ST21 and ST26, while ST31 shared 95\% sequence similarity with ST12, ST13, ST14, ST24, and ST25 (Supplementary Table S1). Sequence similarity was higher in the barcoding region, with 99\% sequence similarity between ST30 and ST21 and ST26 and 98\% similarity between ST31 and ST13, ST14, ST24, ST25, ST21, and ST30 (Supplementary Table S2). On the other hand, pairwise distance comparisons for novel subtypes in the Santin region exhibited greater degrees of divergence than those obtained by analysis of full-length sequences. In the Santin region the highest sequence similarity for ST30 was 93\% with ST21, while ST31 exhibited a 93\% similarity with ST24 and ST25 (Supplementary Table S3). 


\section{Discussion}

Blastocystis is a common parasite of humans, which is also frequently observed in wild and domestic animals [3]. However, the role of wildlife in Blastocystis transmission is not well explored, especially in the United States. Deer are a common wildlife species with habitats that overlap with humans and other domestic and wild animals, which creates the potential for deer to act as reservoirs for pathogens among these populations. Yet, no studies of Blastocystis prevalence or subtype distribution have been conducted in WTD. In the present study, WTD from Maryland, USA were tested for the presence of Blastocystis by PCR and next generation amplicon sequencing, and WTD were found to be commonly infected/colonized with multiple subtypes of this parasite.

Blastocystis was observed in $88.8 \%(71 / 80)$ of the WTD included in this study, indicating that Blastocystis is a common parasite of WTD. Furthermore, Blastocystis occurrence was high in all gender and age categories measured (Table 2). The number of large studies on Blastocystis occurrence in deer are limited, and only two studies have measured Blastocystis occurrence in wild deer populations (Table 1). Of the two previous studies which surveyed Blastocystis in wild deer populations, occurrences of $2 \%$ and $41 \%$ were reported $[29,30]$. These studies were conducted in Australia in red deer [30] and in South Korea in Korean water deer [29], which may contribute to the large difference in occurrence between these studies and the higher occurrence of Blastocytsis in WTD observed in this study. All three studies have targeted different deer species in different geographic locations and used different detection methods to test for the presence of Blastocystis. The high occurrence of Blastocystis in WTD from Maryland, USA and in deer in general indicates that more studies in deer from other regions of the USA and worldwide are needed to better characterize Blastocystis occurrence in deer.

A wide diversity of subtypes was observed in WTD in this study. There were 12 subtypes and 38 unique Blastocystis nucleotide sequences among the 71 Blastocystispositive WTD (Tables 2 and 3). Ten of the subtypes observed in WTD in this study (ST1, ST3, ST4, ST10, ST14, ST21, ST23, ST24, ST25, and ST26) are previously established subtypes $[13,16]$. Of these, only four subtypes (ST1, ST4, ST10, and ST14) have been previously reported in other studies of deer from around the world (Table 1). This is the first study to report ST3 in deer expanding the host range of this subtype. Although ST3 was only observed in two samples, representing $2.8 \%$ of Blastocystis-positive deer, its presence in deer is particularly notable as ST3 is the most common subtype reported in humans [47]. In fact, potentially zoonotic subtypes were present in $8.5 \%$ (6/71) of Blastocystis-positive WTD. The presence of ST3 and two other potentially zoonotic subtypes, ST1 and ST4, in WTD could indicate the potential for zoonosis as well as the possibility of reverse zoonosis between WTD and humans.

Subtypes ST10 and ST14 are two of the most commonly reported subtypes in ruminants (Table 1) and are commonly reported in other studies of deer [3]. ST10 and ST14 were the second and third most common subtypes observed in WTD in $71.8 \%$ and $42.3 \%$ of Blastocystis-positive WTD, respectively. The presence of these two subtypes in WTD further confirms the suitability of ruminants as hosts of ST10 and ST14. To our knowledge, this is the first study to report ST21, ST23, ST24, ST25, and ST26 in deer expanding their host range. These subtypes have been reported in studies of other wild and domestic animals and appear to be prevalent in ruminants $[14,15,24,25,32,48-50]$. The frequent occurrence of these subtypes in WTD in this study further supports the contention that ruminants may be common hosts of these subtypes. Interestingly, ST24 was observed in $77.5 \%$ of Blastocystis-positive WTD making in the most commonly observed subtype in this study. The high occurrence of ST24 in WTD in this study could indicate deer as a potential source of infection for domestic ruminants such as cattle which have lower reported occurrence of ST24 [36,48].

The presence of multiple subtypes of Blastocystis in individual WTD was very common in the study population. Mixed subtype infections were observed in $90.1 \%(64 / 71)$ of Blastocystis-positive WTD in 30 different combinations (Figure 1, Table 2). In the only 
other study to apply NGS to explore Blastocystis subtype diversity in ruminants, mixed infections were also abundant and observed in $65.3 \%$ (49/75) of Blastocystis-positive cattle [48]. NGS has also been used to explore Blastocystis subtype diversity in wild carnivores, humans, captive wild and domestic birds, chickens, wild boars, and pigs with mixed infections reported in $50 \%, 13.7 \%, 62.5 \%, 63.6 \%, 23.1 \%$, and $15.4 \%$ of Blastocystis-positive hosts, respectively $[7,14,15,51,52]$. Thus, the occurrence of mixed subtype infection in WTD reported here is the highest in any Blastocystis host to date. Whether this finding is attributable to WTD physiology or ecology remains to be defined. However, the high occurrence of mixed infections, the high occurrence of Blastocystis overall, and the large number of subtypes present in this wild WTD population could indicate that exposure to multiple sources of infection contribute to infection risk and intra-subtype variability within individual hosts. While lower rates of mixed subtype infections and less subtype diversity in domestic and captive wildlife could indicate shared sources of infection within those populations $[7,14,15,51,52]$.

Multiple sequence variants were observed for nine of the 12 subtypes reported in WTD (Table 3). However, ST10 presented with a markedly large degree of intra-subtype variability compared with other subtypes observed in WTD in this study (Table 3). There were 14 unique sequence variants of ST10, while all other subtypes had between one and three sequence variants. The intra-subtype variability observed in ST10 in WTD is similar to that reported using NGS in cattle where 11 unique ST10 sequence variants were observed among 15 Blastocystis-positive cattle [36]. Unlike cattle, WTD had far less intra-subtype diversity in ST14 and ST24 than might be expected given the common occurrence of these subtypes in WTD. In cattle, there were five unique sequence variants of ST14 reported among 16 positive samples and six unique sequence variants of ST24 among 14 positive samples [36]. While Blastocystis-positive WTD had only three unique sequence variants of ST14 among 30 positive samples and three unique sequence variants of ST24 among 55 positive samples. It is intriguing to speculate that the lack of variability in these two common subtypes of WTD could indicate some degree of host specificity of these sequence variants. Indeed, the three unique sequence variants of ST14 observed in WTD in this study share $99-100 \%$ sequence identity with an ST14 sequence originally reported in a mouflon (Genbank accession\# KC148206) [25]. However, such a conclusion could only be drawn after more extensive sampling and comparison between other domestic and wild ruminant hosts of these subtypes.

There were two novel subtypes observed in WTD in this study, which we propose naming ST30 and ST31. Both novel subtypes were frequently observed, with ST30 in 15.5\% (11/71) of Blastocystis-positive WTD and ST31 in 26.8\% (19/71) of Blastocystis-positive WTD by NGS (Table 3). The NGS protocol used in this study generates sequences of approximately 500 base pairs of a region of the $S S U$ rRNA gene. Recently-proposed guidelines suggest new subtype designations be based on nearly full-length SSU rRNA gene sequences [13]. To achieve fulllength sequences for the two novel subtypes observed in this study, we employed a MinION sequencing strategy which has been demonstrated to be suitable for obtaining high quality full-length Blastocystis reference sequences [15,16,41]. Full-length sequences were obtained for all three sequence variants of ST30 and the single variant of ST31 observed using NGS (Table 3). These sequences were compared to other full-length reference sequences from accepted subtypes of Blastocystis (ST1-ST17, ST21, ST23-ST29) to determine if phylogenetic analysis and pairwise sequence comparison support their designation as new subtypes. Phylogenetic analysis demonstrated strong support for the branching of ST31 with bootstrap support of 94 (Figure 2). Pairwise comparison of full-length sequences also demonstrated that ST31 shares $\leq 95 \%$ sequence similarity with any known subtype (Supplementary Table S1). As such, ST31 clearly meets all recommended criteria for a new subtype designation. ST30 formed a clade with ST21 and ST26, where ST26 branches basally to ST21 and ST30, however, branching within the clade formed by ST21 and ST30 has bootstrap support of 68. Furthermore, pairwise comparisons for ST30 indicate it shares $97 \%$ sequence similarity with ST21 and ST26 (Supplementary Table S1). As ST30 does not clearly fall into any existing subtype category, we suggest it be given a novel subtype designation. This conclusion is further 
supported by the high degree of sequence variance observed for this subtype in the Santin region, where it varies from any named subtype by $\leq 7 \%$ (Supplementary Table S3). Lastly, NGS sequences of ST30 are a near 100\% match to unpublished sequences with no subtype information from sheep from Belgium (Genbank accession \#s HF569206, HF569208, HF569214, and HF569226) indicating that this subtype is found in multiple hosts and geographic regions. There is a clear advantage to providing novel subtypes with a designation that will allow researchers to easily and accurately subtype isolates to understand host specificity and epidemiology.

\section{Conclusions}

This study is the first to use NGS to characterize Blastocystis subtype diversity and occurrence in WTD and the first to study Blastocystis in WTD overall. The common occurrence of Blastocystis in WTD, coupled with the observation of a remarkable number of subtypes, mixed subtype infections, novel subtypes, and zoonotic subtypes in the study population, indicates that WTD and deer in general are an understudied population with potentially important roles in Blastocystis transmission to humans and domestic animals. More studies in deer and other wildlife populations from other regions of the United States and world are needed to understand the role of wildlife in Blastocystis transmission and epidemiology.

Supplementary Materials: The following are available online at https://www.mdpi.com/article/10 $.3390 /$ microorganisms9061343/s1. Supplementary Table S1. Pairwise distances between Blastocystis subtypes full-length SSU rRNA gene sequences showing number of base substitutions per site. Analyses were conducted using the Kimura 2-parameter model and included 70 nucleotide sequences. There were a total of 1950 positions in the final dataset. Supplementary Table S2. Pairwise distances between Blastocystis subtypes barcoding region sequences showing number of base substitutions per site. Analyses were conducted using the Kimura 2-parameter model and included 70 nucleotide sequences. There were a total of 590 positions in the final dataset. Supplementary Table S3. Pairwise distances between Blastocystis subtypes Santin region sequences showing number of base substitutions per site. Analyses were conducted using the Kimura 2-parameter model and included 70 nucleotide sequences. There were a total of 571 positions in the final dataset.

Author Contributions: Conceptualization, J.G.M. and M.S.; Methodology, A.M., N.S.G., J.G.M. and M.S.; Software, A.M. and M.S.; Validation, J.G.M. and M.S.; Formal Analysis, A.M., J.G.M. and M.S.; Investigation, N.S.G., Y.J., A.M., J.G.M. and M.S.; Resources, J.G.M. and M.S.; Data Curation, A.M., J.G.M. and M.S.; Writing-Original Draft Preparation, J.G.M. and M.S.; Writing—Review \& Editing, J.G.M. and M.S.; Visualization, J.G.M. and M.S.; Funding Acquisition, J.G.M. and M.S. All authors have read and agreed to the published version of the manuscript.

Funding: This research was funded by USDA-ARS Project No: 8042-32000-112-00-D.

Institutional Review Board Statement: Not applicable.

Informed Consent Statement: Not applicable.

Data Availability Statement: All relevant data are within the article and its additional files. All raw fastq files were deposited to the NCBI sequence read archive under accession number SRR14607063SRR14607133. The sequences data were submitted to the GenBank database under the accession numbers MZ267636-MZ267679.

Acknowledgments: We thank Phil Norman of the Howard County Department of Recreation and Parks, Md for providing specimens used in this study.

Conflicts of Interest: The authors declare no conflict of interest.

\section{References}

1. El Safadi, D.; Cian, A.; Nourrisson, C.; Pereira, B.; Morelle, C.; Bastien, P.; Bellanger, A.P.; Botterel, F.; Candolfi, E.; Desoubeaux, G.; et al. Prevalence, risk factors for infection and subtype distribution of the intestinal parasite Blastocystis sp. from a large-scale multi-center study in France. BMC Infect. Dis. 2016, 16, 451. [CrossRef]

2. Tito, R.Y.; Chaffron, S.; Caenepeel, C.; Lima-Mendez, G.; Wang, J.; Vieira-Silva, S.; Falony, G.; Hildebrand, F.; Darzi, Y.; Rymenans, L.; et al. Population-level analysis of Blastocystis subtype prevalence and variation in the human gut microbiota. Gut 2019, 68, 1180-1189. [CrossRef] 
3. Hublin, J.S.Y.; Maloney, J.G.; Santin, M. Blastocystis in domesticated and wild mammals and birds. Res. Vet. Sci. 2021, 135, 260-282. [CrossRef]

4. Deng, L.; Wojciech, L.; Gascoigne, N.R.J.; Peng, G.; Tan, K.S.W. New insights into the interactions between Blastocystis, the gut microbiota, and host immunity. PLoS Pathog. 2021, 17, e1009253. [CrossRef]

5. Ajjampur, S.S.R.; Tan, K.S.W. Pathogenic mechanisms in Blastocystis SPP-Interpreting results from in vitro and in vivo studies. Parasitol. Int. 2016, 65, 772-779. [CrossRef] [PubMed]

6. Casero, R.D.; Mongi, F.; Sánchez, A.; Ramírez, J.D. Blastocystis and urticaria: Examination of subtypes and morphotypes in an unusual clinical manifestation. Acta Trop. 2015, 148, 156-161. [CrossRef]

7. Rojas-Velázquez, L.; Maloney, J.G.; Molokin, A.; Morán, P.; Serrano-Vázquez, A.; González, E.; Pérez-Juárez, H.; Ximénez, C.; Santin, M. Use of next-generation amplicon sequencing to study Blastocystis genetic diversity in a rural human population from Mexico. Parasit. Vectors 2019, 12, 566. [CrossRef] [PubMed]

8. Lee, L.; Chye, T.; Karmacharya, B.; Govind, S. Blastocystis SP: Waterborne zoonotic organism, a possibility? Parasit. Vectors 2012, 5, 130. [CrossRef] [PubMed]

9. Caradonna, T.; Marangi, M.; Del Chierico, F.; Ferrari, N.; Reddel, S.; Bracaglia, G.; Normanno, G.; Putignani, L.; Giangaspero, A. Detection and prevalence of protozoan parasites in ready-to-eat packaged salads on sale in Italy. Food Microbiol. 2017, 67, 67-75. [CrossRef] [PubMed]

10. Angelici, M.C.; Nardis, C.; Scarpelli, R.; Ade, P. Blastocystis hominis transmission by non-potable water: A case report in Italy. New Microbiol. 2018, 41, 173-177. [PubMed]

11. Rodrigues, A.C.; da Silva, M.D.C.; Pereira, R.Â.S.; Pinto, L.C. Prevalence of contamination by intestinal parasites in vegetables (Lactuca sativa L. and Coriandrum sativum L.) sold in markets in Belém, Northern Brazil. J. Sci. Food Agric. 2020, 100, $2859-2865$. [CrossRef] [PubMed]

12. Stensvold, C.R.; Suresh, G.K.; Tan, K.S.; Thompson, R.C.; Traub, R.J.; Viscogliosi, E.; Yoshikawa, H.; Clark, C.G. Terminology for Blastocystis subtypes-A consensus. Trends Parasitol. 2007, 23, 93-96. [CrossRef] [PubMed]

13. Stensvold, C.R.; Clark, C.G. Pre-empting Pandora's Box: Blastocystis subtypes revisited. Trends Parasitol. 2020, 36, 229-232. [CrossRef]

14. Maloney, J.G.; Molokin, A.; da Cunha, M.J.R.; Cury, M.C.; Santin, M. Blastocystis subtype distribution in domestic and captive wild bird species from Brazil using next generation amplicon sequencing. Parasite Epidemiol. Control 2020, 9, e00138. [CrossRef] [PubMed]

15. Maloney, J.G.; da Cunha, M.J.R.; Molokin, A.; Cuty, M.C.; Santin, M. Next generation sequencing reveals widespread presence of human-pathogenic Blastocystis subtypes in chickens. Parasitol. Res. 2021, 21, 2219-2231. [CrossRef]

16. Maloney, J.G.; Santin, M. Mind the gap: New full-length sequences of Blastocystis subtypes generated via Oxford Nanopore Minion sequencing allow for comparisons between full-length and partial sequences of the small subunit of the ribosomal RNA gene. Microorganisms 2021, 9, 997. [CrossRef] [PubMed]

17. Stensvold, C.R.; Tan, K.S.W.; Clark, C.G. Blastocystis. Trends Parasitol. 2020, 36, 315-316. [CrossRef]

18. Yan, Y.; Su, S.; Ye, J.; Lai, X.; Lai, R.; Liao, H.; Chen, G.; Zhang, R.; Hou, Z.; Luo, X. Blastocystis sp. subtype 5: A possibly zoonotic genotype. Parasitol. Res. 2007, 101, 1527-1532. [CrossRef]

19. Parkar, U.; Traub, R.J.; Vitali, S.; Elliot, A.; Levecke, B.; Robertson, I.; Geurden, T.; Steele, J.; Drake, B.; Thompson, R.C. Molecular characterization of Blastocystis isolates from zoo animals and their animal-keepers. Vet. Parasitol. 2010, 169, 8-17. [CrossRef]

20. Wang, W.; Owen, H.; Traub, R.J.; Cuttell, L.; Inpankaew, T.; Bielefeldt-Ohmann, H. Molecular epidemiology of Blastocystis in pigs and their in-contact humans in Southeast Queensland, Australia, and Cambodia. Vet. Parasitol. 2014, 203, 264-269. [CrossRef] [PubMed]

21. Greige, S.; El Safadi, D.; Bécu, N.; Gantois, N.; Pereira, B.; Chabé, M.; Benamrouz-Vanneste, S.; Certad, G.; El Hage, R.; Chemaly, M.; et al. Prevalence and subtype distribution of Blastocystis sp. isolates from poultry in Lebanon and evidence of zoonotic potential. Parasit. Vectors 2018, 11, 389. [CrossRef] [PubMed]

22. Köster, P.C.; Dashti, A.; Bailo, B.; Muadica, A.S.; Maloney, J.G.; Santín, M.; Chicharro, C.; Migueláñez, S.; Nieto, F.J.; Cano-Terriza, D.; et al. Occurrence and genetic diversity of protist parasites in captive non-human primates, zookeepers, and free-living sympatric rats in the Córdoba Zoo Conservation Centre, Southern Spain. Animals 2021, 11, 700. [CrossRef]

23. Wang, J.; Gong, B.; Liu, X.; Zhao, W.; Bu, T.; Zhang, W.; Liu, A.; Yang, F. Distribution and genetic diversity of Blastocystis subtypes in various mammal and bird species in northeastern China. Parasit. Vectors 2018, 11, 522. [CrossRef] [PubMed]

24. Zhao, G.H.; Hu, X.F.; Liu, T.L.; Hu, R.S.; Yu, Z.Q.; Yang, W.B.; Wu, Y.L.; Yu, S.K.; Song, J.K. Molecular characterization of Blastocystis SP. in captive wild animals in Qinling Mountains. Parasitol. Res. 2017, 116, 2327-2333. [CrossRef] [PubMed]

25. Alfellani, M.A.; Taner-Mulla, D.; Jacob, A.S.; Imeede, C.A.; Yoshikawa, H.; Stensvold, C.R.; Clark, C.G. Genetic diversity of Blastocystis in livestock and zoo animals. Protist 2013, 164, 497-509. [CrossRef] [PubMed]

26. Oliveira-Arbex, A.P.; David, É.B.; Tenório, M.D.S.; Cicchi, P.J.P.; Patti, M.; Coradi, S.T.; Lucheis, S.B.; Jim, J.; Guimarães, S. Diversity of Blastocystis subtypes in wild mammals from a zoo and two conservation units in southeastern Brazil. Infect. Genet. Evol. 2020, 78, 104053. [CrossRef] [PubMed]

27. Betts, E.L.; Gentekaki, E.; Thomasz, A.; Breakell, V.; Carpenter, A.I.; Tsaousis, A.D. Genetic diversity of Blastocystis in non-primate animals. Parasitology 2018, 145, 1228-1234. [CrossRef] 
28. Betts, E.L.; Gentekaki, E.; Tsaousis, A.D. Exploring micro-eukaryotic diversity in the gut: Co-occurrence of Blastocystis subtypes and other protists in zoo animals. Front. Microbiol. 2020, 11, 288. [CrossRef]

29. Kim, K.T.; Noh, G.; Lee, H.; Kim, S.H.; Jeong, H.; Kim, Y.; Jheong, W.H.; Oem, J.K.; Kim, T.H.; Kwon, O.D.; et al. Genetic diversity and zoonotic potential of Blastocystis in Korean water deer, Hydropotes inermis argyropus. Pathogens 2020, 9, 955. [CrossRef] [PubMed]

30. Roberts, T.; Stark, D.; Harkness, J.; Ellis, J. Subtype distribution of Blastocystis isolates from a variety of animals from New South Wales, Australia. Vet. Parasitol. 2013, 196, 85-89. [CrossRef] [PubMed]

31. Li, X.D.; Zou, Y.; Pan, J.; Liang, Q.L.; Zeng, Z.; Meng, Y.M.; Wang, X.L.; Wang, H.N.; Zhu, X.Q. Prevalence and subtypes of Blastocystis sp. infection in zoo animals in three cities in China. Parasitol. Res. 2020, 119, 465-471. [CrossRef] [PubMed]

32. Stensvold, C.R.; Alfellani, M.A.; Nørskov-Lauritsen, S.; Prip, K.; Victory, E.L.; Maddox, C.; Nielsen, H.V.; Clark, C.G. Subtype distribution of Blastocystis isolates from synanthropic and zoo animals and identification of a new subtype. Int. J. Parasitol. 2009, 39, 473-479. [CrossRef] [PubMed]

33. Ni, H.B.; Gong, Q.L.; Zhang, N.Z.; Zhao, Q.; Tao, W.F.; Qiu, H.Y.; Fei, Y.C.; Zhang, X.X. Molecular detection of Blastocystis in black bears and sika deer in northern China. Parasitol. Res. 2021, 120, 1481-1487. [CrossRef] [PubMed]

34. Li, J.; Karim, M.R.; Li, D.; Rahaman Sumon, S.M.M.; Siddiki, S.H.M.F.; Rume, F.I.; Sun, R.; Jia, Y.; Zhang, L. Molecular characterization of Blastocystis sp. in captive wildlife in Bangladesh National Zoo: Non-human primates with high prevalence and zoonotic significance. Int. J. Parasitol. Parasites Wildl. 2019, 10, 314-320. [CrossRef] [PubMed]

35. Santín, M.; Trout, J.M.; Xiao, L.; Zhou, L.; Greiner, E.; Fayer, R. Prevalence and age-related variation of Cryptosporidium species and genotypes in dairy calves. Vet. Parasitol. 2004, 122, 103-117. [CrossRef] [PubMed]

36. Maloney, J.G.; Molokin, A.; Santin, M. Next generation amplicon sequencing improves detection of Blastocystis mixed subtype infections. Infect. Genet. Evol. 2019, 73, 119-125. [CrossRef]

37. Santín, M.; Gómez-Muñoz, M.T.; Solano-Aguilar, G.; Fayer, R. Development of a new PCR protocol to detect and subtype Blastocystis SPP. from humans and animals. Parasitol. Res. 2011, 109, 205-212. [CrossRef]

38. Bushnell, B. BBMap Download. SourceForge.net. 2014. Available online: https://sourceforge.net/projects/bbmap/ (accessed on 27 April 2020).

39. Rognes, T.; Flouri, T.; Nichols, B.; Quince, C.; Mahé, F. VSEARCH: A versatile open source tool for metagenomics. PEER J. 2016, 4, e2584. [CrossRef]

40. Camacho, C.; Coulouris, G.; Avagyan, V.; Ma, N.; Papadopoulos, J.; Bealer, K.; Madden, T.L. BLAST+: Architecture and applications. BMC Bioinform. 2009, 10, 421. [CrossRef]

41. Maloney, J.G.; Molokin, A.; Santin, M. Use of Oxford Nanopore MinION to generate full-length sequences of the Blastocystis small subunit (SSU) rRNA gene. Parasit. Vectors 2020, 13, 595. [CrossRef]

42. Xiao, L.; Escalante, L.; Yang, C.; Sulaiman, I.; Escalante, A.A.; Montali, R.J.; Fayer, R.; Lal, A.A. Phylogenetic analysis of Cryptosporidium parasites based on the small-subunit rRNA gene locus. Appl. Environ. Microbiol. 1999, 65, 1578-1583. [CrossRef] [PubMed]

43. Koren, S.; Walenz, B.P.; Berlin, K.; Miller, J.R.; Bergman, N.H.; Phillippy, A.M. Canu: Scalable and accurate long-read assembly via adaptive k-mer weighting and repeat separation. Genome Res. 2017, 27, 722-736. [CrossRef] [PubMed]

44. Kimura, M. A simple method for estimating evolutionary rate of base substitutions through comparative studies of nucleotide sequences. J. Mol. Evol. 1980, 16, 111-120. [CrossRef] [PubMed]

45. Kumar, S.; Stecher, G.; Li, M.; Knyaz, C.; Tamura, K. MEGA X: Molecular Evolutionary Genetics Analysis across computing platforms. Mol. Biol. Evol. 2018, 35, 1547-1549. [CrossRef]

46. Scicluna, S.M.; Tawari, B.; Clark, C.G. DNA barcoding of Blastocystis. Protist 2006, 157, 77-85. [CrossRef] [PubMed]

47. Stensvold, C.R.; Clark, C.G. Current status of Blastocystis: A personal view. Parasit. Int. 2016, 65, 763-771. [CrossRef]

48. Maloney, J.G.; Lombard, J.E.; Urie, N.J.; Shively, C.B.; Santin, M. Zoonotic and genetically diverse subtypes of Blastocystis in US pre-weaned dairy heifer calves. Parasitol. Res. 2019, 118, 575-582. [CrossRef] [PubMed]

49. Song, J.K.; Yin, Y.L.; Yuan, Y.J.; Tang, H.; Ren, G.J.; Zhang, H.J.; Li, Z.X.; Zhang, Y.M.; Zhao, G.H. First genotyping of Blastocystis sp. in dairy, meat, and cashmere goats in northwestern China. Acta Trop. 2017, 176, 277-282. [CrossRef]

50. Ren, M.; Song, J.K.; Yang, F.; Zou, M.; Wang, P.X.; Wang, D.; Zhang, H.J.; Zhao, G.H.; Lin, Q. First genotyping of Blastocystis in yaks from Qinghai Province, Northwestern China. Parasit. Vectors 2019, 12, 171. [CrossRef]

51. Calero-Bernal, R.; Santín, M.; Maloney, J.G.; Martín-Pérez, M.; Habela, M.A.; Fernández-García, J.L.; Figueiredo, A.; Nájera, F.; Palacios, M.J.; Mateo, M.; et al. Blastocystis sp. subtype diversity in wild carnivore species from Spain. J. Eukaryot. Microbiol. 2020, 67, 273-278. [CrossRef]

52. Russini, V.; Di Filippo, M.M.; Fanelli, R.; Polidori, M.; Berrilli, F.; Di Cave, D.; Novelletto, A.; Calderini, P. Characterization of prevalence and genetic subtypes of Blastocystis sp. in wild and domestic Suidae of central Italy aided by amplicon NGS. Vet. Parasitol. Reg. Stud. Rep. 2020, 22, 100472. [CrossRef] [PubMed] 